\title{
Internal Orifice Area
}

National Cancer Institute

\section{Source}

National Cancer Institute. Internal Orifice Area. NCI Thesaurus. Code C154711.

The extent of a 2-dimensional surface enclosed within the boundary of an internal orifice. 